\title{
Análise de eventos adversos relacionados com a assistência à saúde
}

\author{
Analysis of adverse events related to health care
}

\author{
Análisis de eventos adversos relacionados con la atención médica
}

Karlla Rodrigues Guerra ${ }^{1 *}$, Fernanda Alves de Lima Gomes ${ }^{1}$, Mayara Carolina Cañedo, Maria Aparecida Munhoz Gaíva².

\section{RESUMO}

Objetivo: Analisar os eventos adversos notificados em um hospital público de capital do centro-oeste brasileiro. Métodos: Trata-se de um estudo transversal retrospectivo, que analisou as notificações enviadas ao Sistema de Notificações para a Vigilância Sanitária, nos anos de 2017 e 2018. Calculou-se a prevalência dos eventos adversos, frequência, médias e coeficiente de Pearson para as demais variáveis estudadas. Resultados: Houve prevalência de eventos adversos em pacientes do sexo masculino, hospitalizados na clínica médica, com idade entre 66 a 75 anos e com média de 10 a 12 dias de internação 0 evento adverso mais frequente foi a Lesão por Pressão; quanto ao tipo de dano, prevaleceu o moderado. O enfermeiro foi o profissional que mais notificou os eventos. Conclusão: Para o melhor controle dos eventos adversos é fundamental o conhecimento do seu perfil e dos pacientes acometidos, visando oferecer subsídios para a gestão da qualidade da assistência e um cuidado mais seguro.

Palavras-chave: Sistema de informação, Segurança do paciente, Assistência à saúde, Doença iatrogênica.

\begin{abstract}
Objective: Objective: To analyze the adverse events reported in the Patient Safety Unit of a public hospital in a capital of Midwestern Brazil . Methods: Methods: This is a descriptive, cross-sectional and retrospective study that analyzed the notifications sent to the Health Surveillance Notification System, in the years 2017 and 2018. The prevalence of the adverse events, frequency, means and Pearson coefficient for the other variables studied have been calculated. Results: There was a prevalence of adverse events in males, in patients hospitalized in the medical clinic, aged between 66 and 75 years and with an average of 10 to 12 days of hospitalization. The most frequent adverse event was Pressure Injury; as for the type of damage, moderate prevailed. The nurse was the professional who most notified the events. Conclusion: For the best control of adverse events, it is essential to know their profile and that of the affected patients, so as to offer subsidies for the management of the quality of care and safer assistance.
\end{abstract}

Keywords: Information system, Patient safety, Delivery of health care, latrogenic disease.

\section{RESUMEN}

Objetivo: Analizar los eventos adversos reportados en un hospital público de la capital del Centro Oeste brasileño. Métodos: Se trata de un estudio transversal retrospectivo, que analizó las notificaciones enviadas al Sistema de Notificación de Vigilancia Sanitaria, en los años 2017 y 2018. Se calculó la prevalencia de eventos adversos, frecuencia, promedios y coeficiente de Pearson para las demás variables estudiadas. Resultados: hubo una prevalencia de eventos adversos en pacientes del sexo masculino, hospitalizados en la clínica médica, con edades entre 66 y 75 años y con un promedio de 10 a 12 días de hospitalización, el

\footnotetext{
${ }^{1}$ Hospital Regional de Mato Grosso do Sul (HRMS), Campo Grande - MS.

*E-mail: maycarolina@hotmail.com

2 Universidade Federal do Mato Grosso (UFMT), Cuiabá - MT.
}

PORTARIA INTERMINISTERIAL MEC/MS № 1.224, DE 03-10-2012 que institui o Programa Nacional de Bolsas para Residências Multiprofissionais. Os resultados apresentados neste estudo referem-se a um recorte do trabalho de Conclusão de Residência. 
evento adverso más frecuente fue la lesión por presión; en cuanto al tipo de daño, predominó el moderado. La enfermera fue la profesional que más notificó los hechos. Conclusión: Para un mejor control de los eventos adversos, es fundamental conocer su perfil y los pacientes afectados, con el objetivo de ofrecer subsidios para la gestión de la calidad de la atención y una atención más segura.

Palabras clave: Sistema de información, Seguridad del paciente, Prestación de atención de salud, Enfermedad iatrogénica.

\section{INTRODUÇÃO}

Incidente relacionado ao cuidado de saúde é um evento que poderia ter resultado, ou resultou, em dano desnecessário ao paciente. Eles são classificados em: near miss, um incidente que não atingiu o paciente; incidente sem dano, evento que atingiu o paciente, mas não causou dano discernível; e incidente com dano, aquele que resulta em dano ao paciente. Assim, o Evento Adverso (EA) é todo incidente com dano ou lesão, que pode ser temporário ou definitivo, e até mesmo causar a morte do paciente (BRASIL, 2017).

Os danos podem ser classificados em: nenhum (o paciente é assintomático e não necessita de tratamento); leve (o paciente é sintomático, porém são sintomas passageiros, nestes os danos são mínimos ou de curta duração, sem intervenção ou com intervenção mínima requerida); moderado (o paciente é sintomático e necessita de intervenção, com aumento nos dias de hospitalização, e os danos são permanentes ou de longo prazo, ou perda de funções); grave (o paciente é sintomático e requer intervenção, pois há risco de morte ou necessidade de intervenção cirúrgica, há diminuição da expectativa de vida ou grandes danos permanentes ou de longo prazo ou ainda perda de funções); e morte (causada ou antecipada pelo incidente) (OMS, 2009).

No ano de 2004, a Organização Mundial da Saúde (OMS) apresentou, por meio da Aliança Mundial para a Segurança do Paciente, diretrizes para um cuidado seguro e de qualidade (OMS, 2008). Estima-se que o EA seja a terceira causa de morte nos Estados Unidos, estando atrás apenas de doenças cardiovasculares e câncer (MAKARY MA e DANIEL M, 2016). Uma revisão integrativa que objetivou evidenciar a produção científica relacionada com a revisão de prontuários para avaliação da incidência e evitabilidade de EA em hospitais, englobando publicações desenvolvidas na Europa, Ásia, África e América do Sul demonstrou que existem diferenças nas taxas de incidência e evitabilidade de EA, as quais variaram de 5,7 a $14,2 \%$ e de 31 a $83 \%$, respectivamente. As divergências se justificam, dentre outros fatores, pela qualidade do cuidado hospitalar prestado em cada continente (ZANETTI ACB, et al., 2020).

No Brasil, um marco importante na assistência à saúde foi a implantação do Programa Nacional de Segurança do Paciente (PNSP), por meio da Portaria GM no 529, de 1ํ de abril de 2013, que tem como objetivo a prevenção e redução da incidência de EA relacionados com a assistência nos serviços de saúde (BRASIL, 2017; BRASIL, 2013). Desde junho de 2014, os EA que ocorrem no país devem ser registrados no Sistema de Notificações para a Vigilância Sanitária (Notivisa), sob responsabilidade da Agência Nacional de Vigilância da Saúde (Anvisa) (MAIA CS, et al., 2018).

No Brasil, cerca de 19,4 milhões de pessoas são hospitalizadas por ano; destas, 1,3 milhão são vítimas de EA por negligência ou imprudência, sendo que 55 mil pessoas morrem por ano devido a um EA, o equivalente a seis pessoas por hora. São diversos os fatores que podem estar envolvidos na ocorrência do EA, dentre eles: a ausência de capacitação para a equipe de saúde, o déficit de recursos humanos, a sobrecarga de trabalho, a comunicação ineficaz e a falta de liderança do gestor (DUARTE SCM, et al., 2015; FIORAVANTI C, 2020; LEMOS GC, et al., 2018).

Um estudo descritivo das notificações dos EA no Notivisa no Brasil, no período de 2014 a 2016, mostrou que foram registrados 63.933 eventos relacionados com a assistência à saúde, sendo que destes, 417 $(0,6 \%)$ evoluíram para óbito (MAIA CS, et al., 2018). Diante do exposto, os EA têm sido considerados importantes indicadores de resultado da qualidade da assistência prestada pelos serviços. Nessa perspectiva, conhecer os eventos, seu gerenciamento, controle e fatores de risco permitem, a toda equipe de saúde e gestores, a implementação de medidas preventivas e tratamentos eficazes. 
No entanto, apesar da importância da notificação dos EA para a segurança dos pacientes, a incidência destes ainda é pouco investigada no país. Assim, este estudo partiu da seguinte pergunta: Quais são as características dos eventos ocorridos em pacientes internados em um hospital público de capital do centrooeste do Brasil? Nessa diretiva, o estudo objetivou analisar os EA notificados em um hospital público de capital do centro-oeste brasileiro.

\section{MÉTODOS}

Trata-se de um estudo transversal retrospectivo, desenvolvido em um hospital público situado na região centro-oeste do Brasil. O hospital em pauta possui 352 leitos e atende pacientes de alta e média complexidade do Sistema Único de Saúde (SUS), em sete linhas de cuidado: cardiovascular, clínica cirúrgica, clínica médica, materno-infantil, nefro-urológica, oncológica e paciente crítico.

Foram avaliados todos os incidentes e os EA enviados ao Notivisa no período de 2017 a 2018, por meio das fichas de notificações voluntárias na plataforma informatizada de gestão hospitalar. O levantamento dos dados foi realizado no primeiro semestre de 2019 , sendo incluídas todas as notificações que ocorreram no hospital no período citado, um total de 898. Foram excluídas as notificações de pacientes não hospitalizados, em duplicidade e as de eventos originados em outros serviços de saúde.

Foram analisadas as seguintes variáveis: idade, sexo, raça/cor e dano. Os danos foram classificados em: sem dano; dano leve; moderado; grave; e morte. As lesões por pressão (LPP) foram classificadas em: estágio I, quando a pele está integra com eritema; estágio II, quando há perda da espessura parcial da pele com exposição da derme; estágio III, perda total da espessura da pele; e estágio IV, perda total da espessura da pele e perda tissular (MORAES JT, 2016). As LPP estágio II são classificadas como dano moderado, já as lesões dos estágios III e IV são consideradas dano grave. As infecções relacionadas com a assistência à saúde (IRAS) são classificadas como danos moderados (BRASIL, 2017).

A prevalência de EA foi calculada dividindo o número de incidentes notificados pelo número de pacientes/dia (população em risco), multiplicado por 100. Para as demais variáveis, foram calculadas médias, percentagens e o coeficiente de correlação de Pearson (R). Todos os cálculos foram realizados no software Microsoft Office Excel® 2007.

A presente pesquisa foi aprovada pelo Comitê de Ética em Pesquisa, protocolo CAAE $n^{\circ}$ 12813919.2.0000.5161 e parecer ํㅜ 3.326.484. Considerando as características da pesquisa, foi solicitada a dispensa do Termo de Consentimento Livre e Esclarecido, mas foram respeitadas todas as recomendações da Resolução 466/2012 do Conselho Nacional de Saúde.

\section{RESULTADOS}

Os resultados demonstraram prevalência de EA em indivíduos do sexo masculino, com $62 \%$ das notificações em 2017 e 59\% das notificações em 2018, com maior ocorrência entre 66 e 75 anos. Por meio das datas de internação e de notificação, calculou-se o tempo médio para ocorrência de EA, sendo que, em 2017, a média foi de 12 dias, e em 2018, de 10 dias após a internação. Houve casos de pacientes que sofreram EA no primeiro dia de internação, e de outros em que o incidente ocorreu após meses e de um relatado após 310 dias de hospitalização.

A prevalência de EA foi de 491 (0,55\%) em 2017 e 407 (0,42\%) em 2018, totalizando 898 incidentes notificados. Observou-se que o EA mais frequente nesta instituição foi a LPP, 283 (57,6\%) em 2017 e 271 (66,6\%) em 2018, seguidas das IRAS, com 95 ocorrências (19,3\%) em 2017 e erro de medicação, com 34 (6,9\%) em 2017. Em 2018 observou-se que o segundo evento de maior ocorrência foi o erro de medicação, com 31 ocorrências $(7,6 \%)$ e o terceiro evento com maior percentual foi a flebite, com 17 notificações $(4,2 \%)$. Ressalta-se que até 2017 as IRAS eram notificadas pelo Serviço de Controle de Infecção Hospitalar (SCIH) e, a partir de 2018, passaram a fazer parte da plataforma informatizada de gestão hospitalar, o que pode justificar o decréscimo no número do evento nesse ano (12/2,9\% dos incidentes), o que pode ser considerada uma subnotificação (Tabela 1). 
Tabela 1 - Distribuição dos eventos adversos notificados no período de 2017 e 2018, segundo o tipo de ocorrência.

\begin{tabular}{|c|c|c|c|c|}
\hline Tipo de ocorrência & (N) 2017 & $(\%)$ & (N) 2018 & $(\%)$ \\
\hline Broncoaspiração & 1 & $0,2 \%$ & 1 & $0,2 \%$ \\
\hline Defeito na qualidade/fabricação de medicação (farmacovigilância) & 0 & $0,0 \%$ & 3 & $0,7 \%$ \\
\hline Dispositivos (invasivos e não invasivos) & 1 & $0,2 \%$ & 0 & $0,0 \%$ \\
\hline Erro de Medicação & 34 & $6,9 \%$ & 31 & $7,6 \%$ \\
\hline Erro/Falha na Hemodiálise & 2 & $0,4 \%$ & 0 & $0,0 \%$ \\
\hline EA com sonda gástrica/enteral & 1 & $0,2 \%$ & 5 & $1,2 \%$ \\
\hline EA com sonda vesical de demora & 6 & $1,2 \%$ & 4 & $1,0 \%$ \\
\hline Exame de Imagens & 5 & $1,0 \%$ & 1 & $0,2 \%$ \\
\hline Exames Laboratoriais e Testes Diagnósticos & 1 & $0,2 \%$ & 6 & $1,5 \%$ \\
\hline Falha na Assistência & 3 & $0,6 \%$ & 1 & $0,2 \%$ \\
\hline Falha na Identificação do paciente & 0 & $0,0 \%$ & 1 & $0,2 \%$ \\
\hline Flebite & 6 & $1,2 \%$ & 17 & $4,2 \%$ \\
\hline Falha relacionada a procedimento cirúrgico & 3 & $0,6 \%$ & 8 & $2,0 \%$ \\
\hline Incidentes com cateter venoso & 16 & $3,3 \%$ & 12 & $2,9 \%$ \\
\hline IRAS & 95 & $19,3 \%$ & 12 & $2,9 \%$ \\
\hline Lesões em recém-nascidos durante o parto & 1 & $0,2 \%$ & 0 & $0,0 \%$ \\
\hline LPP & 283 & $57,6 \%$ & 271 & $66,6 \%$ \\
\hline Óbito (Evento Sentinela) & 1 & $0,2 \%$ & 1 & $0,2 \%$ \\
\hline Outras ocorrências relacionadas a pele & 1 & $0,2 \%$ & 9 & $2,2 \%$ \\
\hline Queda de paciente & 20 & $4,1 \%$ & 11 & $2,7 \%$ \\
\hline Reação adversa & 1 & $0,2 \%$ & 3 & $0,7 \%$ \\
\hline Terapia Nutricional & 5 & $1,0 \%$ & 9 & $2,2 \%$ \\
\hline Transfusão Sanguínea (Hemotransfusão) & 2 & $0,4 \%$ & 1 & $0,2 \%$ \\
\hline Tromboembolismo Venoso (TEV) & 1 & $0,2 \%$ & 0 & $0,0 \%$ \\
\hline TOTAL & 491 & $100,0 \%$ & 407 & $100,0 \%$ \\
\hline
\end{tabular}

Fonte: Guerra KR, et al., 2021; dados extraídos do Notivisa, 2019.

Quanto à classificação dos danos causados pelo EA, prevaleceu o dano moderado com $334(68,1 \%)$ em 2017 e 288 (57,6\%) em 2018 (Figura 1).

Figura 1 - Distribuição do tipo de dano, nos anos de 2017 e 2018.

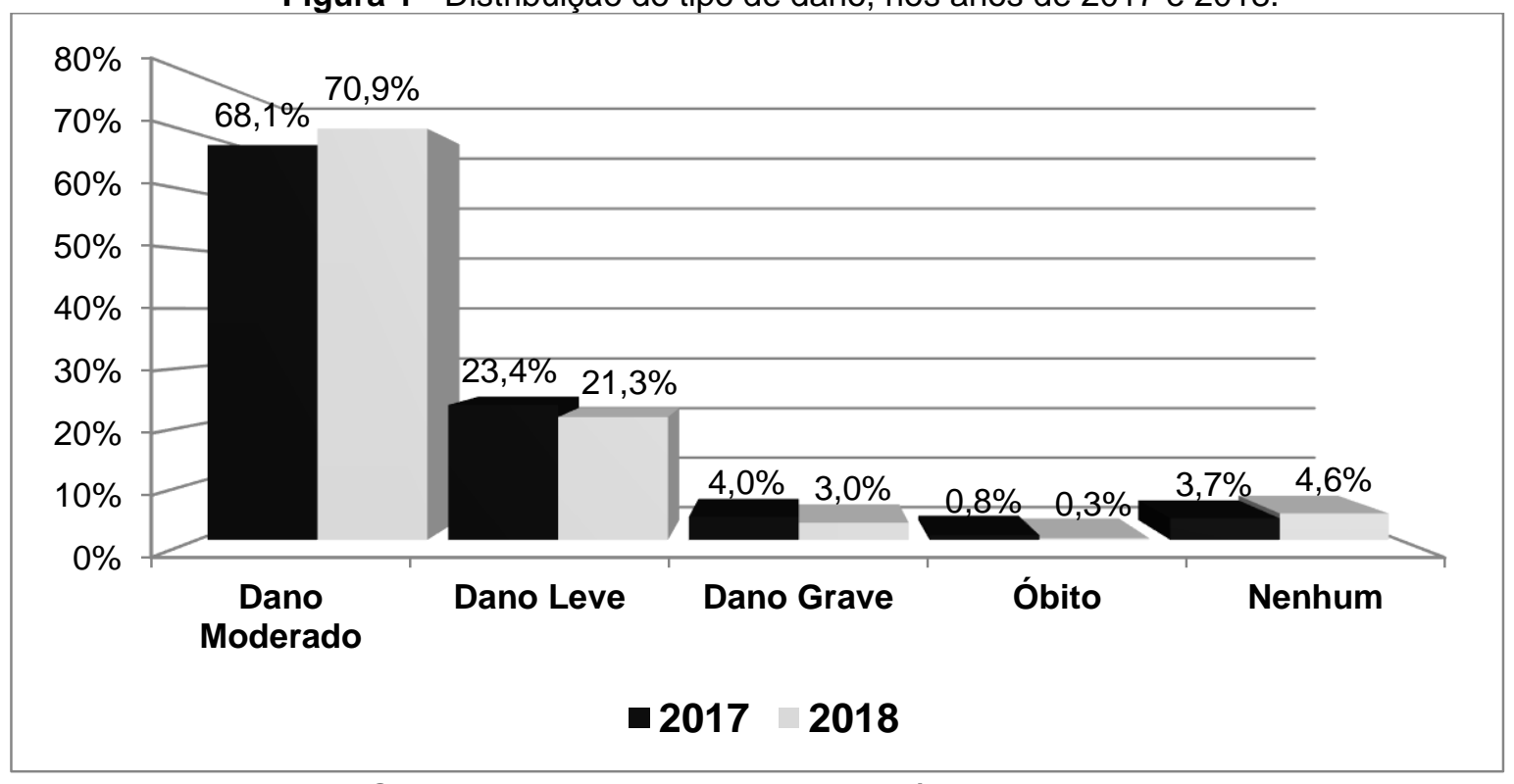

Fonte: Guerra KR, et al., 2021; dados extraídos do Notivisa, 2019. 
Das LPP notificadas, o estágio II foi o mais frequente, sendo $227(46,3 \%)$ no ano de 2017 e $280(68,9 \%)$ em 2018 (Figura 2).

Figura 2 - Percentual do estadiamento de Lesões por Pressão, nos anos de 2017-2018.

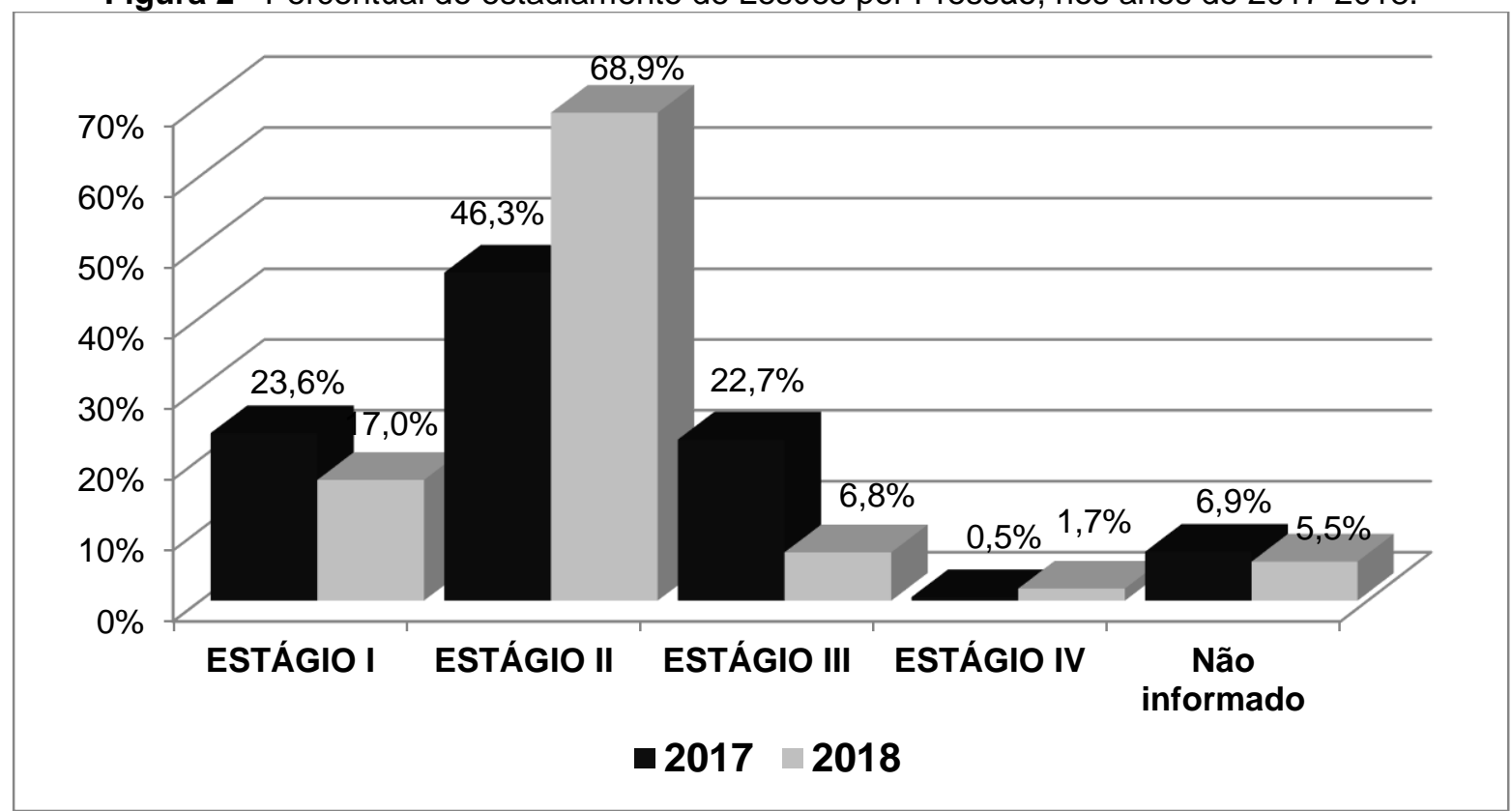

Fonte: Guerra KR, et al., 2021; dados extraídos do Notivisa, 2019.

Das 898 notificações de EA analisadas, a maioria foi comunicada pelo enfermeiro sendo 54,7\% em 2017 e 47,4\% em 2018. O segundo serviço que mais notificou foi a Gerência de Segurança Assistencial e Gestão de Riscos, com 18,5\% dos EA informados em 2017 e 24,1\% em 2018.

Quanto ao local onde ocorreu o EA, no ano de 2017, a clínica médica registrou 158, seguido pelas Unidades de Terapia Intensiva (UTI) adulto com 131. Já no ano de 2018, os setores com maior número de EA foram o Pronto Atendimento Médico (PAM) adulto com 110 e as UTI adulto com 92. Ressalta-se que todos os atendimentos realizados no PAM são registrados em prontuário eletrônico, o que permite a realização da busca ativa dos EA. Os setores com menor número de notificação foram agrupados como outros (Figura 3).

Figura 3 - Distribuição da prevalência dos EA, no ano de 2017, segundo o setor de internação.

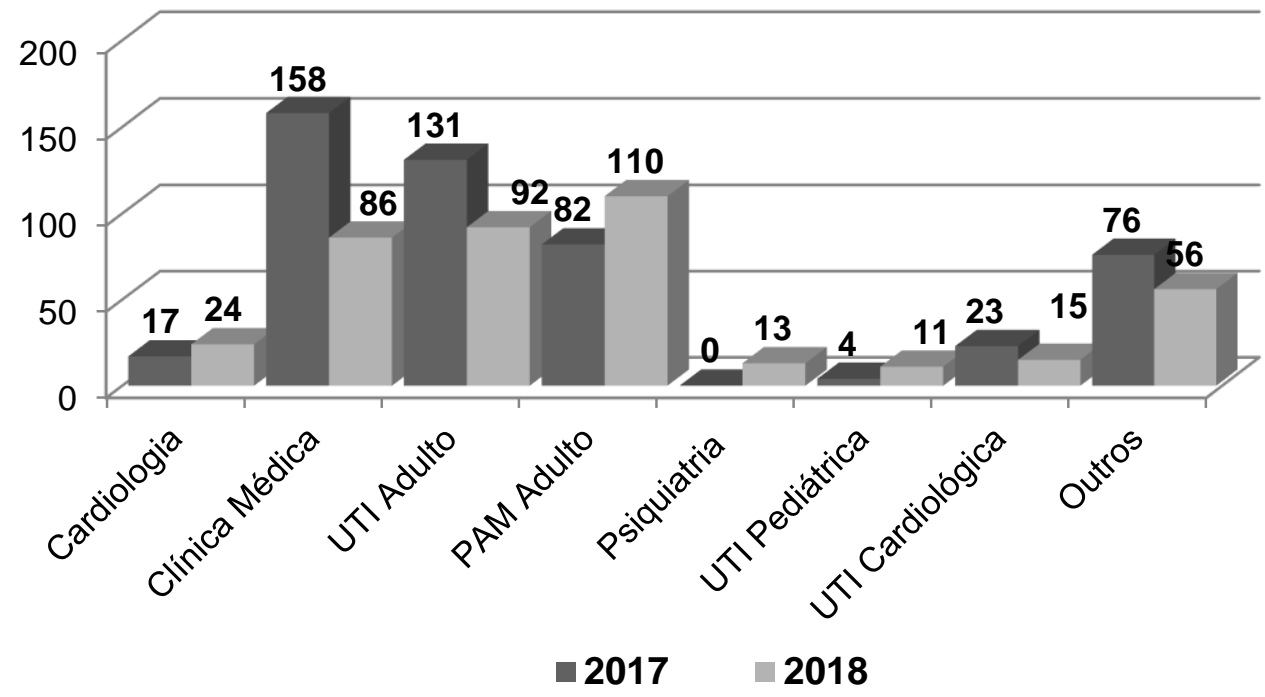

Fonte: Guerra KR, et al., 2021; dados extraídos do Notivisa, 2019. 
Quanto ao turno de ocorrência dos EA, no ano de 2017 o período diurno apresentou a maior prevalência, com $160(78,0 \%)$, enquanto o noturno teve $45(22,0 \%)$; houve significância estatística entre os turnos em que ocorreram os EA $(p<0,001)$. $O$ ano de 2018 seguiu a mesma tendência do ano anterior, sendo o período diurno com 184 notificações (69,4\%), e o noturno com 81 (30,6\%) ( $p<0,001)$ (Tabela 2).

Tabela 2 - Distribuição em número absolutos (n) e relativos (\%) da prevalência de eventos adversos segundo o turno de ocorrência no hospital de estudo.

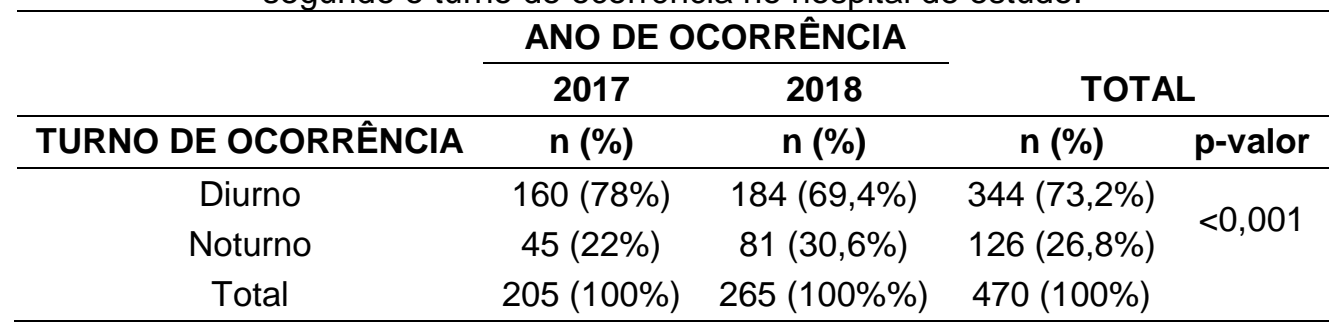

Fonte: Guerra KR, et al., 2021; dados extraídos do Notivisa, 2019.

\section{DISCUSSÃO}

Este estudo possibilitou a análise dos EA notificados ao Núcleo de Segurança do Paciente (NSP) de um hospital público de capital do centro-oeste brasileiro, nos anos de 2017 e 2018. Os EA foram mais prevalentes em pacientes do sexo masculino, idosos, com tempo de ocorrência entre 10 e 12 dias de hospitalização; a LPP estágio II foi o EA mais notificado no período estudado, já o tipo de dano mais frequente foi o moderado. Os EA foram notificados predominantemente pela equipe de enfermagem e 0 setor em que os eventos mais ocorreram foram a clínica médica e a UTI adulto, durante o período diurno.

Os resultados do presente estudo estão em consonância com achados nacionais, pois, segundo o relatório da ANVISA que reúne dados dos EA notificados em todo o território nacional de março de 2014 a junho de 2019, os eventos mais frequentes ocorreram em indivíduos do sexo masculino $(52,3 \%)$ e na faixa etária dos 66 aos 75 anos (BRASIL, 2019). Outro estudo que analisou dados nacionais de notificações de EA com óbitos evidenciou que os adultos a partir de 26 anos e os idosos (60 anos ou mais) representaram a maior parte dos óbitos (85\%), entre os anos de 2014-2016 no país (MAIA CS, et al., 2018).

Nos países desenvolvidos, onde há uma cultura de segurança em saúde mais disseminada, a prevalência de EA em pacientes hospitalizados gira em torno de 4,0 a 16,0\%. Ao comparar essas taxas com as encontradas no hospital em estudo (0,55\% em 2017 e 0,42\% em 2018), é perceptível uma cultura de segurança pouco desenvolvida, demonstrando ainda que há subnotificação (BRASIL, 2017). Recomenda-se que o NSP priorize ações de melhoria na comunicação entre os profissionais, assim como a disseminação da cultura de segurança na instituição para que os trabalhadores tenham mais adesão à notificação dos EA.

No que se refere ao tempo de internação para a ocorrência do EA, a análise de 193 casos em hospital de referência em cardiologia no estado de Santa Catarina, Brasil, encontrou resultados semelhantes aos desta pesquisa, em que a maioria dos eventos ocorreu entre 0 a 10 dias $(22,6 \%)$ da internação do paciente (LANZONI GMM, et al., 2019). Em outro estudo brasileiro, a mediana de intervalo de tempo entre a data da internação e a data do incidente foi de quatro dias (MAIA CS, et al., 2018).

Observou-se neste estudo que a LPP foi o EA mais notificado, semelhante aos dados nacionais informados ao Notivisa entre janeiro de 2014 a julho de 2017, em que dos 134.501 incidentes notificados, $23.722(17,6 \%)$ corresponderam às LPP, o terceiro tipo de evento mais notificado no país (BRASIL, 2017).

A alta incidência de notificações de LPP é preocupante, visto que, no II Anuário de Segurança Assistencial Hospitalar no Brasil (2018), a incidência de óbitos foi $20 \%$ mais alta em pacientes expostos há pelo menos um dos EA graves, entre eles, endocardite infecciosa, choque séptico, LPP (estágio III ou IV), insuficiência respiratória aguda, dentre outros (COUTO RC, et al., 2018). A prevenção da LPP é uma das metas do PNSP, e sua responsabilidade recai sobre a equipe multidisciplinar em todos os níveis de atenção à saúde. Além disso, existem evidências de que a maioria das LPP é evitável durante o processo de hospitalização (BRASIL, 2017). 
Investigação realizada em hospital universitário da Tunísia com 150 pacientes de 4 até 86 anos, internados por período prolongado e com risco de LPP, evidenciou que 29 deles apresentaram LPP, e destes, 20 (69\%) em estágio IV, diferentemente desta pesquisa em que predominou o estágio II. Os autores ressaltam que a incidência de LPP impõe maior necessidade de intervenções, tanto aquelas voltadas ao paciente quanto ao sistema de saúde, devido ao aumento da carga de trabalho da equipe de saúde e a demanda financeira (GHALI H, et al., 2018)

Pesquisa retrospectiva de análise de EA em prontuários de hospital privado de Natal, Rio Grande do Norte, mostrou que das 262 notificações ocorridas em 2015 e 2016, os principais EA evitáveis foram os erros de administração de medicamentos, 116 (44,27\%); falha durante a digitação da prescrição médica, 46 $(17,56 \%)$; e falha durante assistência à saúde, 35 (13,36\%). Ocorreram também 19 (7,25\%) quedas; duas $(0,76 \%)$ falhas durante procedimento cirúrgico; duas $(0,76 \%)$ LPP e uma $(0,38 \%)$ falha na administração de dietas (NETO AVL, 2019). Já no presente estudo, as LPP foram os EA mais notificados, seguidos pela IRAS em 2017 e erros de medicação em 2018.

Um estudo de coorte realizado nas unidades de clínica médica e cirúrgica de um hospital do interior do Rio Grande do Sul, cujo objetivo era avaliar o risco para quedas de pacientes adultos hospitalizados e verificar a incidência do EA, evidenciou, durante o acompanhamento de 831 participantes durante 122 dias, que 19 caíram, uma média de 4,7 quedas ao mês. Calculando-se o índice de queda por pessoa/dia, no total de 6.400 pacientes/dia, a taxa de incidência de queda foi de 1,68\% (PASA TS, et al., 2017). Os dados apresentados no presente estudo demonstram que há uma subnotificação das taxas de quedas no hospital analisado, pois no ano de 2017 foram notificadas 20 quedas e, em 2018, apenas 11.

As IRAS podem ser desenvolvidas durante a internação ou após a alta hospitalar (BRASIL, 2016). Estudo realizado com 75 pacientes em um hospital de Manaus, Amazonas, Brasil, que caracterizou os casos de IRAS em uma UTI adulta, identificou o predomínio das infecções relacionadas com o trato respiratório em $46,2 \%$ e com a corrente sanguínea em 26,6\% deles (HESPANHOL LAB, et al., 2019). Por sua vez, pesquisa relacionada a infecções de corrente sanguínea envolvendo 3.402 observações de administração de medicamentos endovenosos em acesso venoso central, em uma UTI adulta no estado de Sergipe, demonstrou a baixa adesão dos profissionais à prática de higienização das mãos e à desinfecção de materiais, injetores e conectores (OLIVEIRA JKA, et al., 2017). No presente estudo, as IRAS foram o segundo EA mais notificado em 2017, com 95 ocorrências. No entanto não foram registrados os tipos de IRAS.

Quanto às notificações de EA por erro de medicação observou-se nesta pesquisa um aumento nos dois anos estudados, respectivamente $(6,9 \%$ e $7,6 \%)$. Revisão integrativa que avaliou as estratégias utilizadas pela equipe de enfermagem para minimizar os erros de medicação nas unidades de emergência mostrou que, dentre estas, estão as estratégias educacionais, mudanças organizacionais, como implementação de protocolos e o uso de novas tecnologias como a prescrição informatizada, implantação da dose unitária e do código de barras na administração de medicamentos (OLIVEIRA EBC, et al., 2019).

Em relação ao grau de dano, houve predomínio de dano leve e moderado, com poucos casos de dano grave, achado semelhante aos encontrados em estudo realizado em hospital público do Distrito Federal, Brasil, que analisou os EA no período de 2011 a 2014 (GÖTTEMS LBB, et al., 2016). Sabe-se que os EA graves, além de aumentarem o tempo médio de permanência dos pacientes nas instituições hospitalares, majoram os custos financeiros da saúde (COUTO RC, et al., 2018).

Quanto à categoria profissional notificadora, pesquisa realizada em um complexo hospitalar universitário do interior do estado de São Paulo também mostrou que os enfermeiros foram os profissionais que mais notificaram (71\%), enquanto que os médicos contribuíram com $8 \%$ das notificações (FURINI ACA, et al., 2019). Uma revisão integrativa que analisou as publicações nacionais sobre os motivos para a não notificação de EA por profissionais nos serviços de saúde brasileiros, apontou também os enfermeiros como os principais responsáveis pela notificação de EA (ALVES, MFT et al., 2019). Esses achados podem ter relação com o fato de os enfermeiros permanecerem mais próximos dos pacientes, além de estarem 
acostumados com as demandas administrativas, como o preenchimento das fichas de notificações. Todavia, ressalta-se que a responsabilidade pela segurança do paciente deveria ser compartilhada igualmente por todos os membros da equipe de saúde.

Pesquisa descritiva que analisou as notificações de EA que tiveram como dano o óbito entre os anos de 2014 e 2016 no Brasil identificou que os setores que requerem maior utilização de tecnologias e procedimentos invasivos têm maior predisposição à incidência de EA, como as UTI (MAIA CS, et al., 2018). Neste estudo, tanto em 2017 como em 2018, a clínica médica e a UTI adulto foram os setores que tiveram maior número de notificações de EA. Sabe-se que nas unidades de clínica médica, a grande maioria dos pacientes internados são idosos e com alta dependência para realizar as atividades da vida diária.

Quanto ao período ou turno de trabalho em que ocorreu o EA, estudo baseado nos dados do gerenciamento de risco de um complexo hospitalar localizado no noroeste paulista identificou que as ocorrências foram mais prevalentes no período diurno. Os autores relacionam esse resultado ao fato de que nesse período se concentram a realização do maior número de procedimentos e atendimentos ambulatoriais (FURINI ACA, et al., 2019). Resultado que corrobora os achados desta pesquisa, já que a maioria dos EA notificados ocorreu no período diurno. No hospital em pauta as atividades ambulatoriais como coleta de exames laboratoriais e de imagem e quimioterápicos são realizadas no período diurno. Além disso, os banhos dos pacientes acamados são priorizados para o turno matutino, o que facilita a visualização das LPP, o EA mais notificado no serviço.

As pesquisas em diferentes contextos brasileiros mostram que ainda ocorre subnotificação dos EA, seja por desconhecimento de sua importância, seja pela falta de acesso aos documentos necessários ou, ainda, pelo medo de repreensões (NEGRÃO SMC, et al., 2019). Ainda há baixo conhecimento dos profissionais de saúde sobre os EA e como notificá-los, além do medo da equipe em expor os erros cometidos, devido às políticas de punição nas instituições de saúde. Sabe-se da importância da adoção nos serviços de uma cultura de segurança que permita à equipe informar as ocorrências, sem medo de ações punitivas que responsabilizem apenas o profissional, mas que identifiquem também as fragilidades no sistema, uma vez que o conhecimento dos EA possibilita a adoção de medidas preventivas eficazes (ALVES MFT, et al., 2019; DUARTE SCM, et al., 2015; BRASIL, 2013).

Apesar de o presente estudo ter utilizado dados secundários, que podem ter falhas de preenchimento e dubiedade de informações, que afetam a sua qualidade, os resultados são relevantes para o hospital, já que o estudo foi uma etapa inicial para investigar os EA no serviço. Para estudos futuros sugere-se avaliação da cultura de segurança no referido hospital, utilizando instrumentos já testados em nosso país e que podem contribuir para a promoção de cuidados seguros na instituição.

\section{CONCLUSÃO}

Para o melhor controle dos EA é fundamental o conhecimento do seu perfil e o dos pacientes acometidos, visando oferecer subsídios para a gestão da qualidade da assistência e um cuidado mais seguro. Sabe-se que os EA podem ser reduzidos pela adoção de intervenções que minimizem o risco, com o intuito de evitar os incidentes cujos resultados implicam em prejuízos físicos, emocionais e financeiros para o paciente, para a equipe de saúde diretamente envolvida em seu cuidado e para a instituição. Apesar das notificações de EA estarem centradas no enfermeiro, vale ressaltar que elas não são de responsabilidade de uma única categoria profissional, sendo necessária a sensibilização da equipe multiprofissional para que tenham maior envolvimento com a segurança do paciente.

\section{AGRADECIMENTOS E FINANCIAMENTO}

Agradecemos a bolsa recebida do Programa de Residência Multiprofissional Integrado em Saúde, área de concentração Intensivismo, do Hospital Regional de Mato Grosso do Sul e ao Ministério da Saúde que, por meio da portaria interministerial MEC/MS № 1.224, de 03-10-2012, instituiu o Programa Nacional de Bolsas para Residências Multiprofissionais. 


\section{REFERÊNCIAS}

1. ALVES MFT, et al. Motivos para a não notificação de incidentes de segurança do paciente por profissionais de saúde: revisão integrativa. Ciência \& Saúde Coletiva. 2019; 24(8): 2895-2908.

2. BRASIL. Agência Nacional de Vigilância Sanitária. Relatório nacional de incidentes relacionados à assistência à saúde. Boletim Segurança do Paciente e Qualidade em Serviços de Saúde.2017. Disponível em: https://www20.anvisa.gov.br/segurancadopaciente/index.php/publicacoes/category/boletins-estatisticos. Acesso em: 20 novembro 2019.

3. BRASIL. Agência Nacional de Vigilância Sanitária. Assistência Segura: Uma Reflexão Teórica Aplicada à Prática Agência Nacional de Vigilância Sanitária. Brasília: Anvisa, 2017. Disponível em: http://www.saude.pi.gov.br/uploads/divisa_document/file/374/Caderno_1_-Assist\%C3\%AAncia_Segura_-
Uma Reflex\%C3\%A30_Te\%C3\%B3rica_Aplicada \%C3\%A0_Pr\%C3\%A1tica.pdf. Acessado em: 23 fevereiro Uma

4. BRASIL. Agência Nacional de Vigilância Sanitária. Gestão de Riscos e Investigação de Eventos Adversos Relacionados à Assistência à Saúde. Brasília: Anvisa, 2017. Disponível em: https://portaldeboaspraticas.iff.fiocruz.br/biblioteca/gestao-de-riscos-e-investigacao-de-eventos-adversosrelacionados-a/. Acessado em: 20 novembro 2019.

5. BRASIL. Agência Nacional de Vigilância Sanitária. Práticas seguras para prevenção de Lesão por Pressão em serviços de saúde. NOTA TÉCNICA GVIMS/GGTES № 03/2017. Disponível em: https://www20.anvisa.gov.br/segurancadopaciente/index.php/alertas/item/nota-tecnica-gvims-ggtes-03-2017. Acessado em: 20 novembro 2019.

6. BRASIL. Agência Nacional de Vigilância Sanitária. Relatórios dos estados no período de janeiro de 2014 a maio de 2019. Disponível em: < https://www20.anvisa.gov.br/segurancadopaciente/index.php/publicacoes/category/relatoriosdos-estados>. Acessado em: 20 novembro 2019.

7. BRASIL. Portaria no 529, de 10 de abril de 2013. Institui o Programa Nacional de Segurança do Paciente (PNSP). Diário Oficial da União n. 62, seção 1, p72, 31.05.2013.

8. BRASIL. Programa Nacional de Prevenção e Controle de Infecções Relacionadas à Assistência à Saúde (20162020). Gerência Geral de Tecnologia em Serviços de Saúde - GGTES Brasília, novembro de 2016. Disponível em: https://www.saude.go.gov.br/images/imagens_migradas/upload/arquivos/2017-02/pnpciras-2016-2020.pdf. Acessado em: 20 novembro 2019 .

9. COUTO RC, et al. II Anuário da segurança assistencial hospitalar no Brasil: propondo as prioridades nacionais. Instituto de estudos de saúde suplementar. 2018. Disponível em: https://www.iess.org.br/cms/rep/Anuario2018.pdf. Acessado em: 20 novembro 2019.

10. DUARTE SCM, et al. Eventos adversos e segurança na assistência de enfermagem. Revista Brasileira de Enfermagem. 2015, 68(1): 144-154.

11. FIORAVANTI C. Um diagnóstico do Erro Médico. Saúde Pública, Revista Pesquisa FAPESP, 2020; p.59-61.

12. FURINI ACA, et al. Notificação de eventos adversos: caracterização dos eventos ocorridos em um complexo hospitalar. Revista Gaúcha de Enfermagem. 2019; 40(esp): e20180317.

13. GHALI H, et al. Incidence and risk factors of pressure ulcers in a Tunisian university hospital. Revue d'Epidémiologie et de Santé Publique. 2018; 66(Supl 5): S340.

14. GÖTTEMS LBD, et al. Estudo dos casos notificados como incidentes em um hospital público de 2011 a 2014. Revista da Escola de Enfermagem da USP. 2016; 50(5): 863-869.

15. HESPANHOL LAB , et al. Infecção relacionada à Assistência à Saúde em Unidade de Terapia Intensiva Adulto. Enfermería Global. 2019; 53: 229-241.

16. LANZONI GMM, et al. Eventos adversos e incidentes sem danos em unidades de internação de um hospital especializado em cardiologia. Revista Mineira de Enfermagem. 2019; 23: e1184.

17. LEMOS GC, et al. A cultura de segurança do paciente no âmbito da enfermagem: reflexão teórica. Revista de Enfermagem do Centro-Oeste Mineiro. Revista de Enfermagem do Centro-Oeste Mineiro. 2018; 8: e2600

18. MAIA CS, et al. Notificações de eventos adversos relacionados com a assistência à saúde que levaram a óbitos no Brasil, 2014-2016. Epidemiologia Serviço de Saúde. 2018; 27(2): e2017320.

19. MAKARY M, DANIEL M. Medical Error - The third leading cause of death in the US. British Medical Journal. 2016; 353: $2139-2154$.

20. MORAES JT, et al. Conceito e classificação de lesão por pressão: atualização do National Pressure Ulcer Advisory Panel. Revista de Enfermagem do Centro-Oeste Mineiro. 2016; 6(2): 2292-2306.

21. NEGRÃO SMC, et al. Avaliação da prática de enfermagem na segurança do paciente oncológico. Enfermagem em Foco. 2019; 10 (4): 136-142

22. NETO AVL, et al. Análisis de las notificaciones de eventos adversos en un hospital privado. Enfermeria Global. 2019; 18(3): 314-343.

23. OLIVEIRA EBC, et al. Estratégias para minimizar erros de medicação em unidades de emergência: revisão integrative. Revista Brasileira de Enfermagen [Internet]. 2019; 72(Suppl 1): 320-327.

24. OLIVEIRA JKA. Patient safety in nursing care during medication administration. Revista Latino-Americana de Enfermagem. 2018; 26: e3017.

25. PASA TS, et al. Risk assessment and incidence of falls in adult hospitalized patients. Revista Latino-Americana de Enfermagem. 2017; 25: e2862.

26. WHO - World Health Organization. Conceptual Framework for the International Classification for Patient Safety: Final Technical Report [Internet]. Geneva: WHO; 2009 Disponível em: http://www.who.int/patientsafety/taxonomy/icps_full_report.pdf. Acessado em: 23 fevereiro 2021.

27. WHO - World Health Organization. World Alliance for Patient Safety. Forward programme 2008-2009. Geneva: WHO, 2008. WHO - http://www.who.int/patientsafety/information_centre/reports/Alliance_Forward_Programme_2008.pdf. Acessado em: 23 fevereiro 2021.

28. ZANETTI ACB, et al. Avaliação da incidência e evitabilidade de eventos adversos em hospitais: revisão integrativa. Revista Gaúcha de Enfermagem. 2020; 41: e20190364. 